\title{
Universal Broadening of the Bardeen-Cooper-Schrieffer Coherence Peak of Disordered Superconducting Films
}

\author{
M. V. Feigel'man and M. A. Skvortsov \\ L. D. Landau Institute for Theoretical Physics, 142432 Chernogolovka, Russia and \\ Moscow Institute of Physics and Technology, 141700 Moscow, Russia
}

(Dated: June 19, 2018)

\begin{abstract}
In disordered superconductors, the local pairing field fluctuates in space, leading to the smearing of the BCS peak in the density of states and appearance of the subgap tail states. We analyze the universal mesoscopic contributions to these effects and show that they are enhanced by the Coulomb repulsion. In the vicinity of the quantum critical point, where superconductivity is suppressed by the "fermionic mechanism", strong smearing of the peak due to mesoscopic fluctuations is predicted.

PACS numbers: 74.78.-w, 74.20.-z, 74.81.-g
\end{abstract}

Superconductive ( $s$-wave) state is characterized by a gap $\Delta$ in the quasiparticle spectrum and the coherence peak $(\mathrm{CP})$ in the density of states (DOS) above the gap, $\rho(E)=\rho_{0} \operatorname{Re} E / \sqrt{E^{2}-\Delta^{2}}$. According to classical results [1, 2], impurity scattering does not affect this picture, as long as the time-reversal invariance (TRI) is not broken. Yet, a number of experiments demonstrate considerable suppression of the $\mathrm{CP}$ and appearance of subgap $(E<\Delta)$ states with the increase of disorder $[3-$ 5]. A mechanism leading to gap smearing without invoking any TRI breaking was proposed 40 years ago in the seminal paper [6] (see also Ref. 7). It was shown that the effect of a (phenomenologically introduced) short-scale disorder in the Cooper attraction constant, $\lambda=\bar{\lambda}+\delta \lambda(\mathbf{r})$, is formally equivalent to the one produced by magnetic impurities [8]. Another mechanism [9] relates smearing of the $\mathrm{CP}$ with a finite inelastic lifetime of quasiparticles [10]; this effect becomes exponentially weak at low temperatures, $T \ll T_{c}$. Finally, it has been recently demonstrated that an apparent DOS smearing seen in tunnelling experiments may be due to electric fluctuations in the environment [11].

In recent few years an upsurge of interest in experimental studies of strongly disordered (non-granular) superconductors has been seen, evidenced, e. g., by Refs. 12 16]. Two basic classes of these materials distinguished by the value of the electron concentration are known (for a review, see Ref. 17]). Below we will focus on strongly disordered superconductors with high (typical metallic) electron density and strong Coulomb interaction [13, 15, 16], where the fermionic mechanism of superconductivity suppression by disorder 18] is operating.

In this Letter we show that mesoscopic conductance fluctuations [19] provide a universal lower bound for the DOS smearing effects in any disordered superconducting thin films, effective down to $T=0$. For the case of thin films (thickness $d$ is below the low-temperature coherence length $\xi_{0}$ ), the strength of this smearing is completely controlled by the film dimensionless conductance $g=2 \pi \hbar / e^{2} R_{\square} \gg 1$, and the critical conductance, $g_{c}=\ln ^{2}\left(\hbar / T_{c 0} \tau_{*}\right) / 2 \pi$, for the fermionic mechanism of su- perconductivity suppression 18]. Here $T_{c 0}$ is the transition temperature in the clean system (we put Boltzmann constant $\left.k_{B}=1\right)$ and $\tau_{*}=\max \left\{\tau, \tau(d / l)^{2}\right\}$, where $\tau$ is the elastic scattering time and $l=v_{F} \tau$ is the mean free path.

We find that the average DOS schematically shown in Fig. 11is characterized by two energy scales: The width $\Gamma$ measures the broadening of the BCS peak, while $\Gamma_{\text {tail }}$ determines the exponential decay rate of the subgap DOS, $\langle\rho(E)\rangle \propto \exp \left\{-\left[\left(E_{g}-E\right) / \Gamma_{\text {tail }}\right]^{3 / 2}\right\}$. The tail in the averaged DOS is a manifestation of the local gap inhomogeneity due to randomness in impurities' configuration. In the zero-temperature limit, $\Gamma_{\text {tail }}$ and $\Gamma \gg \Gamma_{\text {tail }}$ are given by

$$
\frac{\Gamma_{\text {tail }}}{\Delta_{0}}=\left[\frac{0.47}{g\left(g-g_{c}\right)}\right]^{2 / 3}, \quad \frac{\Gamma}{\Gamma_{\text {tail }}}=\left(\ln \frac{\Delta_{0}}{\Gamma_{\text {tail }}}\right)^{2 / 3} .
$$

The most important feature of the result (10) is a sharp growth of the DOS broadening in the vicinity of the quantum critical point, $g=g_{c}$. The same dimensionless parameter, $\delta_{d} \sim 1 / g\left(g-g_{c}\right)$, is known to control the disorder-induced smearing of the thermal transition [20].

Our quantitative analysis is presented below.

Mean-field structure of the superconducting state.-We start the analysis of the superconducting state at the mean-field level, working with disorder-averaged quantities and neglecting spatial fluctuations of the order parameter. The effect of the Coulomb interaction on the properties of disordered superconducting films is usually treated in terms of an energy-dependent Cooper amplitude $\lambda(\zeta)$, with $\zeta=\ln \left(1 / E \tau_{*}\right)$ being the logarithm of the running energy scale (hereafter we set $\hbar=1$ ). In the case of the screened Coulomb interaction, $\lambda(\zeta)$ obeys the RG equation [18]:

$$
d \lambda / d \zeta=\lambda^{2}-\lambda_{g}^{2}, \quad \lambda_{g}^{2}=1 / 2 \pi g,
$$

where we neglected the triplet sector contribution and conductance renormalization assuming $\zeta / g \ll 1$. Equation (2) describes a competition between Cooper instability and Coulomb suppression of Cooper attraction, with 


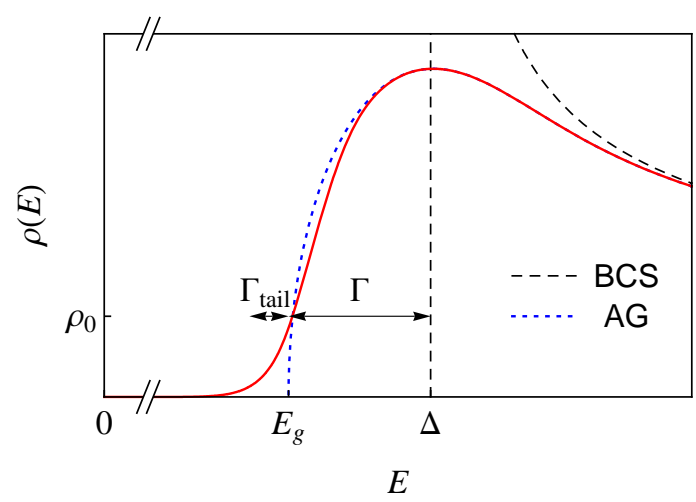

FIG. 1: (color online). Schematic view of the average DOS in a dirty superconducting film (solid line). Broadening of the BCS peak (dashed line) is mainly described by the semiclassical approximation (dotted line), with the full DOS containing a significant tail of the subgap states.

the initial condition $\lambda(0)=\lambda_{0}$ at $E \sim \tau_{*}^{-1}$. The RG flow (2) drives $\lambda(\zeta)$ to infinity at $\zeta_{*}=\left(2 \lambda_{g}\right) \ln \left[\left(\lambda_{0}+\lambda_{g}\right) /\left(\lambda_{0}-\right.\right.$ $\left.\left.\lambda_{g}\right)\right]$, which signals the superconducting transition with the critical temperature $T_{c}=\tau_{*}^{-1} e^{-\zeta_{*}}[18]$ :

$$
\frac{T_{c} \tau_{*}}{\hbar}=\left(\frac{\sqrt{g}-\sqrt{g_{c}}}{\sqrt{g}+\sqrt{g_{c}}}\right)^{\sqrt{\pi g / 2}} .
$$

Another important feature introduced by the Coulomb interaction is the energy dependence of the pairing potential $\tilde{\Delta}(\varepsilon)$ [20]. The latter is defined through the quasiclassical Gorkov function in the Matsubara representation, $F(\varepsilon)=\tilde{\Delta}(\varepsilon) /\left[\varepsilon^{2}+\tilde{\Delta}^{2}(\varepsilon)\right]^{1 / 2}$. The function $\tilde{\Delta}(\varepsilon)$ can be obtained from the self-consistency equation (SCE)

$$
\tilde{\Delta}(\varepsilon)=\pi T \sum_{\varepsilon^{\prime}} \lambda\left(\varepsilon, \varepsilon^{\prime}\right) F\left(\varepsilon^{\prime}\right)
$$

where $\varepsilon$ is the fermionic Matsubara energy, and the energy-dependent Cooper amplitude is given by

$$
\lambda\left(\varepsilon, \varepsilon^{\prime}\right)=\lambda_{0}-\lambda_{g}^{2} \ln \left[1 / \max \left(\varepsilon, \varepsilon^{\prime}\right) \tau_{*}\right] .
$$

A description based on Eqs. (4) and (5), where all energies are retained, is alternative to successive elimination of high-energy degrees of freedom by the RG evolution of $\lambda(\zeta)$. The logarithmic correction to $\lambda_{0}$ in Eq. (5) corresponds to the last term in RG equation (2). At the same time, the first term in Eq. (2) is automatically taken into account by the summation over energy in Eq. (4). An approach based on Eqs. (4) and (5) is equivalent to the RG in the determination of $T_{c}$, but can be used also to find $\tilde{\Delta}(\varepsilon)$ in the superconducting phase at $T<T_{c}$.

Equation (44) generalizes the SCE in the BCS theory. To find the energy dependence of $\tilde{\Delta}(\varepsilon)$ at large energies, $\varepsilon \gtrsim T_{c}$, we rewrite Eq. (4) as a linear integral equation in terms of the logarithmic variable $\zeta$, which is readily solved by reducing to a differential equation owing to a simple form of the kernel (5). As a result, we arrive at

$$
\tilde{\Delta}(\varepsilon)=\Delta(T)\left[\left(\varepsilon / T_{c}\right)^{\lambda_{g}}+\left(\varepsilon / T_{c}\right)^{-\lambda_{g}}\right] / 2,
$$

valid for $\varepsilon \gtrsim T_{c}$. Here $\Delta(T)$ is a function of temperature which should be determined from the full equation (44), where the region of small energies, $\varepsilon \sim T_{c}$, becomes important. Neglecting a slow $\varepsilon$-dependence of $\tilde{\Delta}(\varepsilon)$, we conclude that $\Delta(T)$ is related to $T_{c}$ as in the standard BCS theory [in particular, $\Delta(0)=1.76 T_{c}$ ] .

According to Eq. (6), high-energy $\left(E \gg T_{c}\right)$ electrons experience a larger value of the effective pairing potential 20]. This effect is most pronounced in the limit of strong suppression of superconductivity, $T_{c} \ll T_{c 0}$, when the overall enhancement becomes large: $\tilde{\Delta}\left(\tau_{*}^{-1}\right) \gg \Delta(T)$.

Smearing by inhomogeneities. - The mean-field theory developed above describes disorder-averaged quantities. In the presence of a quenched disorder, the order parameter becomes non-uniform and the sharp BCS peak gets broadened. Analytical description of this effect is complicated due to the failure of the perturbation theory at $E \rightarrow \Delta$. Earlier experience [6, 21] suggests that the problem can be conveniently tackled in two steps:

1. First one has to find the correlation function $\left\langle\Delta(\mathbf{r}) \Delta\left(\mathbf{r}^{\prime}\right)\right\rangle$. Since this is a thermodynamic quantity involving contributions from many energies, it can be obtained in the Matsubara representation by a regular perturbation theory.

2. Then behavior of electrons in the field of a spatially fluctuating $\Delta(\mathbf{r})$ can be considered independently for each real energy $E$.

This scheme based on the perturbation theory is applicable provided that disorder smearing is small.

In dirty superconductors, diffusive motion of electrons is described by the Usadel equation [22] supplemented by the SCE (4). Various types of disorder, such as magnetic impurities [8, 23] or fluctuating coupling constant [6, 21], can be easily incorporated into the scheme as random fields in the Usadel equation. The situation with universal mesoscopic disorder (intrinsic fluctuations of the potential disorder) we are considering is different: Since the Usadel equation is already written for the ensembleaveraged quantities, mesoscopic potential disorder cannot be included there as some extra fluctuating field. To find the correlation function $\left\langle\Delta(\mathbf{r}) \Delta\left(\mathbf{r}^{\prime}\right)\right\rangle$, one has to go beyond the Usadel equation, consider two replicas of the system and average over soft diffusive modes [20], similar to calculation of the universal conductance fluctuations (UCF) [19].

Mesoscopic fluctuations of the order parameter.-In the presence of disorder, the SCE (4) contains two sources of disorder: mesoscopic fluctuations of the coupling constant, $\lambda_{\text {dis }}\left(\varepsilon, \varepsilon^{\prime} ; \mathbf{r}\right)$ (in its Coulomb part), and mesoscopic fluctuations of the Gorkov function, $F_{\text {dis }}(\varepsilon ; \mathbf{r})$. 
These quantities exhibit fluctuations even for a uniform order parameter since they are governed by diffusive motion of electrons sensitive to mesoscopic disorder. Due to the SCE, this fluctuations will result in an inhomogeneous contribution to the order parameter: $\tilde{\Delta}(\varepsilon ; \mathbf{r})=\tilde{\Delta}(\varepsilon)+\tilde{\Delta}_{1}(\varepsilon ; \mathbf{r})$. The latter, in turn, will modify $F$ which therefore can be represented in the form $F=F_{0}+\left(\partial F_{0} / \partial \tilde{\Delta}\right) \tilde{\Delta}_{1}+F_{\text {dis }}$. Substituting this in the SCE (4) and linearizing we get an equation for $\tilde{\Delta}_{1}(\varepsilon)$ in the Fourier representation:

$$
\begin{aligned}
& \tilde{\Delta}_{1}(\varepsilon, \mathbf{q})-\pi T \sum_{\varepsilon^{\prime}} \lambda\left(\varepsilon, \varepsilon^{\prime}\right) \frac{\partial F_{0}\left(\varepsilon^{\prime}, \mathbf{q}\right)}{\partial \tilde{\Delta}\left(\varepsilon^{\prime}, \mathbf{q}\right)} \tilde{\Delta}_{1}\left(\varepsilon^{\prime}, \mathbf{q}\right) \\
& =\pi T \sum_{\varepsilon^{\prime}}\left[\lambda\left(\varepsilon, \varepsilon^{\prime}\right) F_{\mathrm{dis}}\left(\varepsilon^{\prime}, \mathbf{q}\right)+\lambda_{\mathrm{dis}}\left(\varepsilon, \varepsilon^{\prime}, \mathbf{q}\right) F_{0}\left(\varepsilon^{\prime}\right)\right] .
\end{aligned}
$$

In the $2 \mathrm{D}$ case, the main contribution to the $\mathrm{CP}$ smearing comes from large scales [see Eq. [13)], much exceeding the correlation length $r_{c} \sim \xi_{0} \sim \sqrt{D / T_{c}}$ for mesoscopic fluctuations of $F_{\text {dis }}$ and $\lambda_{\text {dis }}$. Therefore it suffices to consider $\tilde{\Delta}_{1}(\varepsilon)$ at zero momentum which will be implied below.

Inverting the matrix in the left-hand side of Eq. (7), we express $\tilde{\Delta}_{1}(\varepsilon)$ in terms of $F_{\text {dis }}$ and $\lambda_{\text {dis. }}$. To study the CP smearing we need the small-energy limit of $\tilde{\Delta}_{1}(\varepsilon)$ with $\varepsilon \sim T_{c}$, where the effect of fluctuations is to modify $\Delta(T) \rightarrow \Delta(T)+\delta \Delta(T)$, with $\delta \Delta(T)$ given by [24]

$$
\begin{aligned}
\delta \Delta(T)= & L_{0}\left(\frac{T}{T_{c}}\right) \frac{(2 \pi T)^{2}}{\Delta(T)} \sum_{\varepsilon_{1}, \varepsilon_{2}>0} F_{0}\left(\varepsilon_{1}\right) \\
& \times\left[\lambda\left(\varepsilon_{1}, \varepsilon_{2}\right) F_{\mathrm{dis}}\left(\varepsilon_{2}\right)+\lambda_{\mathrm{dis}}\left(\varepsilon_{1}, \varepsilon_{2}\right) F_{0}\left(\varepsilon_{2}\right)\right] .
\end{aligned}
$$

Here $L_{0}$ is the fluctuation propagator at zero momentum and frequency in the BCS theory:

$$
L_{0}^{-1}\left(\frac{T}{T_{c}}\right)=\pi T \sum_{\varepsilon} \frac{\Delta^{2}(T)}{\mathfrak{E}^{3}}, \quad \mathfrak{E}=\sqrt{\varepsilon^{2}+\Delta^{2}(T)},
$$

with the asymptotic behavior $L_{0} \approx 4 \pi^{2} T^{2} / 7 \zeta(3) \Delta^{2}(T)$ at $T \rightarrow T_{c}$, and $L_{0}=1$ at $T=0$.

Therefore, smearing of the $\mathrm{CP}$ and behavior near the gap edge are determined by a single number, $f(0)=$ $\langle\delta \Delta \delta \Delta\rangle_{\mathbf{q}=0}$, which can be easily obtained from the correlation functions of $F_{\text {dis }}$ and $\lambda_{\text {dis }}$ with the help of Eq. (8). Since the Coulomb correction to $\lambda$ already contains a closed loop (return probability), the correlation functions $\left\langle F_{\text {dis }} F_{\text {dis }}\right\rangle,\left\langle F_{\text {dis }} \lambda_{\text {dis }}\right\rangle$ and $\left\langle\lambda_{\text {dis }} \lambda_{\text {dis }}\right\rangle$ are given by the one-, two- and three-loop diagrams in soft diffusive modes, respectively. The overall contribution is given by [24]

$$
f(0)=\frac{\pi D \Delta(T)}{g\left(g-g_{c}\right)} K\left(\frac{T}{T_{c}}\right),
$$

where $K\left(T / T_{c}\right)=L_{0}^{2}\left(T / T_{c}\right) N\left(T / T_{c}\right)$ (see Fig. 2), and

$$
N\left(\frac{T}{T_{c}}\right)=16 T^{2} \sum_{\varepsilon_{1}, \varepsilon_{2}>0} \frac{\Delta(T)}{\mathfrak{E}_{1} \mathfrak{E}_{2}\left(\mathfrak{E}_{1}+\mathfrak{E}_{2}\right)},
$$

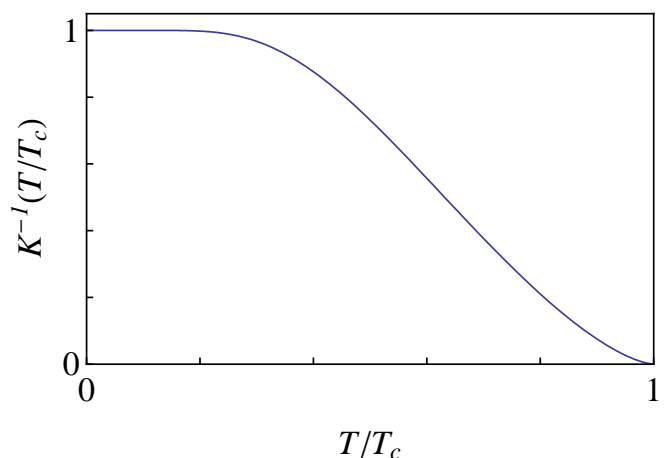

FIG. 2: (color online). Plot of the function $K^{-1}\left(T / T_{c}\right)$, where $K(t)=L_{0}^{2}(t) N(t)$ is defined by Eqs. (9) and (11).

with $N \approx 14 \zeta(3) \Delta(T) / \pi^{3} T$ at $T \rightarrow T_{c}$, and $N=1$ at $T=0$. Replacement of $1 / g^{2}$ by $1 / g\left(g-g_{c}\right)$ in Eq. (10) is due to high-energy contributions, $T_{c}<\varepsilon<\tau_{*}^{-1}$, where $\tilde{\Delta}(\varepsilon)$ is enhanced according to Eq. (6). In the limit of strong $T_{c}$ suppression, $g-g_{c}<g_{c}$, the leading source of disorder comes from mesoscopic fluctuations of the return probability in $\left\langle\lambda_{\text {dis }} \lambda_{\text {dis }}\right\rangle$. Equation (10) is consistent with our previous result in the vicinity of $T_{c}$ [20], generalizing it to arbitrary temperatures $T<T_{c}$.

Mean-field density of states.-The average DOS, $\langle\rho(E)\rangle=\rho_{0} \operatorname{Re}\langle\cos \theta(E, \mathbf{r})\rangle$, is expressed in terms of the spectral angle $\theta$ which satisfies the Usadel equation, $(D / 2) \nabla^{2} \theta+i E \sin \theta+\Delta(\mathbf{r}) \cos \theta=0$, with a random order parameter, $\Delta(\mathbf{r})=\Delta_{0}+\delta \Delta(\mathbf{r})$ [here $\Delta_{0} \equiv \Delta(T)$ ]. Integrating out short-range degrees of freedom one gets an equation for the long-range behavior of $\theta(E, \mathbf{r})[\underline{6}, 21]$ :

$$
\frac{D}{2} \nabla^{2} \theta+i E \sin \theta+\Delta(\mathbf{r}) \cos \theta-\Delta_{0} \eta \cos \theta \sin \theta=0
$$

where the depairing strength is expressed in terms of the correlation function $f(\mathbf{q})=\langle\delta \Delta \delta \Delta\rangle_{\mathbf{q}}$ as

$$
\eta=\frac{2}{\Delta_{0}} \int \frac{f(\mathbf{q})}{D q^{2}} \frac{d^{2} \mathbf{q}}{(2 \pi)^{2}} .
$$

This expression has been originally derived in Ref. [6] for the 3D geometry. The last term in Eq. (12) coincides with the depairing term due to magnetic impurities derived by Abrikosov and Gorkov (AG) 8]. It leads to the broadening of the $\mathrm{CP}$ (shown by the dotted line in Fig. 1), with the hard gap at $E_{g}^{\mathrm{AG}}=\left(1-\eta^{2 / 3}\right)^{3 / 2} \Delta_{0}$.

In the marginal 2D case, mapping to the problem of magnetic impurities should be done with care. Contrary to the 3D geometry, now the integral in Eq. (13) is logarithmically divergent at small $q$. An appropriate cutoff can be established by retaining the Cooperon mass in Eq. (13): $D q^{2} \mapsto D q^{2}+D / L_{E}^{2}$, with $D / L_{E}^{2}=$ $2\left(-i E \cos \theta+\Delta_{0} \sin \theta+\Delta_{0} \eta \cos 2 \theta\right)$. Thus, in the $2 \mathrm{D}$ geometry, $\eta$ becomes a function of $\theta$ and $E$, making the depairing term in the Usadel equation more complicated 
than the simple AG term. However since the dependence of $\eta$ on $L_{E}$ is logarithmically slow, we can evaluate it at the AG solution replacing $\theta$ by $\theta_{\mathrm{AG}}(E)$ :

$$
L_{E}=\frac{D^{1 / 2}}{\left[24 \Delta_{0}\left(E_{g}-E\right)\right]^{1 / 4}} \sim \xi(T)\left(\frac{E_{g}}{E_{g}-E}\right)^{1 / 4}
$$

(we assume $\eta \ll 1$ ). As a result, the depairing factor becomes energy-dependent:

$$
\eta(E)=\frac{K\left(T / T_{c}\right)}{g\left(g-g_{c}\right)} \ln \frac{\min \left(L_{E}, L_{g}\right)}{\xi_{0}},
$$

where we had to introduce an infrared length scale $L_{g}$ to regularize the otherwise divergent $\eta\left(E \rightarrow E_{g}\right)$. Its appearance is related to the breakdown of the mean-field approximation in the narrow region $\left|E-E_{g}\right| \lesssim \Gamma_{\text {tail }}$ [6], where proliferation of instantons generates a finite correlation length $L_{g} \sim \xi(T)\left(E_{g} / \Gamma_{\text {tail }}\right)^{1 / 4}$. Substituting $\eta=\eta\left(E_{g}\right)$ into $E_{g}^{\mathrm{AG}}$, we obtain for $\Gamma \equiv \Delta(T)-E_{g}$ :

$$
\frac{\Gamma}{\Delta(T)}=\frac{3}{2}\left[\frac{K\left(T / T_{c}\right)}{4 g\left(g-g_{c}\right)} \ln \frac{L_{g}(T)}{\xi_{0}}\right]^{2 / 3},
$$

which in the zero-temperature limit reduces to Eq. (11).

The theory developed above applies to quasi-2D films with the thickness $d \ll \xi_{0}$. For finite $d \lesssim \xi_{0}$, there exists a contribution to $\eta$ coming from short scales $(l \ll r \ll d)$ where electron diffusion is $3 \mathrm{D}[24]$ :

$$
\eta_{3 \mathrm{D}}=\frac{2 \Delta(T)}{\pi^{2} \hbar D}\left(\frac{\rho}{R_{Q}}\right)^{2} \ln \frac{d}{l} \sim \frac{d^{2}}{\xi^{2}(T)} \frac{1}{g^{2}} \ln \frac{d}{l},
$$

where $\rho$ is the film resistivity, $R_{Q}=2 \pi \hbar / e^{2}$, and $\xi(T)$ is the temperature-dependent coherence length. Correction (17) which should be added to Eq. (15) leads to a small increase of the width $\Gamma$.

Subgap states.-A hard gap in the excitation spectrum predicted by the AG theory is smeared by disorder leading to the formation of states at $E<E_{g}$. These localized states are identified as instantons in the nonlinear equation (12) 6, 21, 23, 25]. In Ref. 6], Lifshitz-type arguments were used to determine an optimal fluctuation of $\Delta(\mathbf{r})$ in Eq. (12) giving rise to a finite DOS at $E<E_{g}$. On the contrary, in Ref. [21], Eq. (12) was considered for a uniform $\Delta(\mathbf{r})=\Delta_{0}$, and instantons related with intrinsic nonlinearity of the Usadel equation were analyzed. The results of Refs. [6] and [21] are different and represent two asymptotics of a unique function of $E_{g}-E$ (a detailed discussion will be given elsewhere [26]). For small $E_{g}-E$, nonlinearity is weak and the subgap DOS is due to optimal fluctuations of $\Delta(\mathbf{r})[\underline{6}$, whereas the mechanism of Ref. 21] is applicable only for very large $E_{g}-E$, where the DOS is exponentially small.

Generalizing the 3D analysis of Ref. [6] to the 2D case [24], we find that the DOS decays exponentially in the subgap region:

$$
\begin{gathered}
\langle\rho(E)\rangle \propto \exp \left\{-\left[\left(E_{g}-E\right) / \Gamma_{\text {tail }}\right]^{3 / 2}\right\}, \\
\frac{\Gamma_{\text {tail }}}{\Delta(T)}=\left[0.47 \frac{K\left(T / T_{c}\right)}{g\left(g-g_{c}\right)}\right]^{2 / 3}
\end{gathered}
$$

[in the $d$-dimensional space, $\left.\ln \langle\rho(E)\rangle \propto-\left(E_{g}-E\right)^{2-d / 4}\right]$. At $T=0$, Eq. (19) reduces to Eq. (11). Equation (18) is valid as long as $E_{g}-E>\Gamma_{\text {tail }}$. Note that the instanton action obtained in Ref. [21] neglecting $\Delta(\mathbf{r})$ fluctuations is extremely large, $S \sim g$, already at $E_{g}-E \sim \Gamma_{\text {tail }}$. Hence, $\langle\rho(E)\rangle$ follows Eq. (18) for all conceivable $E<E_{g}$.

Discussion.-Superconducting samples always have some amount of disorder which leads to smearing of the BCS density of states. We have considered the case of a minimal possible disorder — intrinsic randomness in a homogeneously disordered film responsible for the UCF in the normal state. The average DOS sketched in Fig. 11 is characterized by two energy scales. The shift of the gap edge $\Delta_{0} \rightarrow E_{g}$ and related suppression of the $\mathrm{CP}$ height is controlled by the parameter $\Gamma$, so that $\rho_{\max }(E) / \rho_{0} \approx(\Delta / \Gamma)^{1 / 2}$. At the same time, the width of the subgap tail is determined by a different parameter $\Gamma_{\text {tail }}<\Gamma$. Both $\Gamma$ and $\Gamma_{\text {tail }}$ are small in a clean system but get enhanced as the film becomes less conductive approaching the critical point, $g \rightarrow g_{c}$. Smearing of the DOS structure becomes very strong at $g-g_{c} \sim 1 / g_{c}$, where our theory becomes inapplicable. The CP broadening is temperature dependent and grows at $T \rightarrow T_{c}$ due to the growth of the function $K\left(T / T_{c}\right)$ (see Fig. 2).

Our results for the disorder-induced DOS smearing should be compared with the smearing due to inelastic scattering [9]. In a $2 \mathrm{D}$ system, the inelastic rate due to Coulomb interaction is of the order of $\Gamma_{e e} \sim T / g$ at $T \sim$ $T_{c}$, and gets exponentially suppressed, $\Gamma_{e e} \propto e^{-\Delta(T) / T}$, at $T \ll T_{c}$ [10]. Therefore our mechanism always dominates at low temperatures. It also always dominates close to $T_{c}$, where $K\left(T / T_{c}\right) \propto\left(1-T / T_{c}\right)^{-3 / 2}$. The general relation between the rates $\Gamma$ and $\Gamma_{e e}$ depends on the proximity to the quantum critical point. Relatively far from it, at $g-g_{c} \gtrsim \sqrt{g}_{c}$, inelastic scattering is the leading source of the DOS smearing at intermediate temperatures, $T_{1} \lesssim T \lesssim T_{2}$, where $T_{1} \sim \Delta_{0} / \ln \beta$ and $T_{c}-T_{2} \sim \beta^{-2}$, with $\beta=\left(g-g_{c}\right)^{2 / 3} / g^{1 / 3}$. For films closer to criticality, $g-g_{c} \lesssim \sqrt{g}_{c}$, disorder-induced smearing always dominates over the inelastic smearing.

Developed theory is expected to be most appropriate for very thin films of amorphous metallic superconductors (e.g., Mo-Ge, Nb-Si, W-Re, $\mathrm{Nb}_{3} \mathrm{Ge}$ ), where suppression of $T_{c}$ by disorder is described by the fermionic mechanism [18], and $R_{\square} \geq 1-2 \mathrm{k} \Omega$.

Finally, we emphasize that the widely used phenomenological Dynes ansatz, $\rho(E)=\rho_{0} \operatorname{Re}(E-i \Gamma) /[(E-$ $\left.i \Gamma)^{2}-\Delta^{2}\right]^{1 / 2}[3]$ is inapplicable when disorder is the main source of the broadening. The actual DOS profile then 
depends on two energy parameters, $\Gamma$ and $\Gamma_{\text {tail }}$, and decays exponentially rather than algebraically at $E<E_{g}$.

We are grateful to I. S. Burmistrov, T. M. Klapwijk, and A. Silva for useful discussions. This work was supported by the RFBR grant No. 10-02-01180, the Dynasty Foundation, and the Russian Federal Agency of Education (contract No. P799) (M. S.).

[1] A. A. Abrikosov and L. P. Gor'kov, Zh. Eksp. Teor. Fiz. 35, 1558 (1958); 36, 319 (1959) [Sov. Phys. JETP 8, 1090 (1959); 9, 220 (1959)].

[2] P. W. Anderson, J. Phys. Chem. Solids 11, 26 (1959).

[3] R. C. Dynes, V. Narayanamurti, and J. P. Garno, Phys. Rev. Lett. 41, 1509 (1978); R. C. Dynes, J. P. Garno, G. B. Hertel and T. P. Orlando, Phys. Rev. Lett. 53, 2437 (1984).

[4] H. Tashiro et al., Phys. Rev. B 78, 014509 (2008).

[5] G. C. O'Neil et al., J. Low Temp. Phys. 151, 70 (2008).

[6] A. I. Larkin and Yu. N. Ovchinnikov, Zh. Eksp. Teor. Fiz. 61, 2147 (1971) [Sov. Phys. JETP 34, 1144 (1972)].

[7] R. Oppermann, Z. Phys. B 63, 33 (1986).

[8] A. A. Abrikosov and L. P. Gor'kov, Zh. Eksp. Teor. Fiz. 39, 1781 (1960) [Sov. Phys. JETP 12, 1243 (1961)].

[9] D. A. Browne, K. Levin and K. A. Muttalib, Phys. Rev. Lett. 58, 156 (1987).

[10] T. P. Devereaux and D. Belitz, Phys. Rev. B, 44, 4587 (1991).

[11] J. P. Pekola, V. F. Maisi, S. Kafanov et al., Phys. Rev.
Lett. 105, 026803 (2010).

[12] B. Sacepe, T. Dubouchet, C. Chapelier et al., Nature Phys. 7, 239 (2011).

[13] B. Sacepe, C. Chapelier, T. Baturina et al., Nature Commun. 1, 140 (2010).

[14] O. Astafiev, L. Ioffe, S. Kafanov et al., Nature 484, 355 (2012).

[15] A. Pourret, H. Aubin, J. Lesueur et al., Nature Phys. 2, 683 (2006); Phys. Rev. B 76, 214504 (2007).

[16] V. Mourik, K. Zuo, S. M. Frolov, S. R. Plissard, E. P. A. M. Bakkers, and L. P. Kouwenhoven, Science 336, 1003 (2012).

[17] M. Feigel'man, L. Ioffe, V. Kravtsov and E. Cuevas, Ann. Phys. (NY) 325, 11390 (2010).

[18] A. M. Finkestein, Pis'ma Zh. Eksp. Teor. Fiz. 45, 37 (1987) [Sov. Phys. JETP Lett. 45, 46 (1987)]; Physica B 197, 636 (1994).

[19] B. L. Altshuler, Pis'ma Zh. Eksp. Teor. Fiz. 41, 530 (1985) [Sov. Phys. JETP Lett. 41, 648 (1985)]; P. A. Lee and A. D. Stone, Phys. Rev. Lett. 55, 1622 (1985).

[20] M. A. Skvortsov and M. V. Feigel'man, Phys. Rev. Lett. 95, 057002 (2005).

[21] J. S. Meyer and B. D. Simons, Phys. Rev. B 64, 134516 (2001).

[22] K. Usadel, Phys. Rev. Lett. 25, 507 (1970).

[23] A. Lamacraft and B. D. Simons, Phys. Rev. Lett. 85, 4783 (2000); Phys. Rev. B 64, 014514 (2001).

[24] See Supplemental Material.

[25] P. M. Ostrovsky, M. A. Skvortsov, M. V. Feigel'man, Phys. Rev. Lett. 87, 027002 (2001).

[26] M. A. Skvortsov and M. V. Feigel'man, to be published. 


\section{Supplemental Material}

\section{INVERSION OF THE FLUCTUATION PROPAGATOR}

Here we invert the matrix fluctuation propagator defined by the left-hand side of Eq. (7):

$$
\tilde{\Delta}_{1}(\varepsilon)-\pi T \sum_{\varepsilon^{\prime}} \lambda\left(\varepsilon, \varepsilon^{\prime}\right) \frac{\partial F_{0}\left(\varepsilon^{\prime}\right)}{\partial \tilde{\Delta}\left(\varepsilon^{\prime}\right)} \tilde{\Delta}_{1}\left(\varepsilon^{\prime}\right)=\phi(\varepsilon) .
$$

Evaluating the derivative of $F\left(\varepsilon^{\prime}\right)=\tilde{\Delta}\left(\varepsilon^{\prime}\right) /\left[\varepsilon^{\prime 2}+\tilde{\Delta}^{2}\left(\varepsilon^{\prime}\right)\right]^{1 / 2}$, we get

$$
\tilde{\Delta}_{1}(\varepsilon)-\pi T \sum_{\varepsilon^{\prime}} \lambda\left(\varepsilon, \varepsilon^{\prime}\right) F_{0}\left(\varepsilon^{\prime}\right) \frac{\tilde{\Delta}_{1}\left(\varepsilon^{\prime}\right)}{\tilde{\Delta}\left(\varepsilon^{\prime}\right)}+\pi T \sum_{\varepsilon^{\prime}} \frac{\lambda\left(\varepsilon, \varepsilon^{\prime}\right) \tilde{\Delta}^{2}\left(\varepsilon^{\prime}\right)}{\left[\varepsilon^{\prime 2}+\tilde{\Delta}^{2}\left(\varepsilon^{\prime}\right)\right]^{3 / 2}} \tilde{\Delta}_{1}\left(\varepsilon^{\prime}\right)=\phi(\varepsilon) .
$$

Since both $\tilde{\Delta}\left(\varepsilon^{\prime}\right)$ and $\tilde{\Delta}_{1}\left(\varepsilon^{\prime}\right)$ are logarithmically slow functions of $\varepsilon^{\prime}$, the second sum can be easily evaluated and we arrive at

$$
\tilde{\Delta}_{1}(\varepsilon)-\pi T \sum_{\varepsilon^{\prime}} \lambda\left(\varepsilon, \varepsilon^{\prime}\right) F_{0}\left(\varepsilon^{\prime}\right) \frac{\tilde{\Delta}_{1}\left(\varepsilon^{\prime}\right)}{\tilde{\Delta}\left(\varepsilon^{\prime}\right)}+L_{0}^{-1} \lambda\left(\varepsilon, T_{c}\right) \tilde{\Delta}_{1}\left(T_{c}\right)=\phi(\varepsilon),
$$

where $L_{0}$ is the fluctuation propagator at zero frequency and momentum in the BCS theory:

$$
L_{0}^{-1}\left(\frac{T}{T_{c}}\right)=\pi T \sum_{\varepsilon} \frac{\Delta^{2}(T)}{\left[\varepsilon^{2}+\Delta^{2}(T)\right]^{3 / 2}}= \begin{cases}\frac{7 \zeta(3) \Delta^{2}(T)}{4 \pi^{2} T^{2}}, & T_{c}-T \ll T_{c} \\ 1, & T \ll T_{c} .\end{cases}
$$

The value of $\tilde{\Delta}_{1}\left(T_{c}\right)$ can be easily obtained from Eq. (S22). In order to do this we multiply it by $F(\varepsilon)$ and sum over $\varepsilon$. Using the SCE (4), we immediately see that the first two terms in Eq. (S22) cancel and we obtain

$$
\tilde{\Delta}_{1}\left(T_{c}\right)=\frac{L_{0}}{\Delta(T)} \pi T \sum_{\varepsilon} F_{0}(\varepsilon) \phi(\varepsilon),
$$

where we use that, according to Eq. (6), $\tilde{\Delta}\left(T_{c}\right)=\Delta(T)$.

\section{CORRELATION FUNCTION $\left\langle\Delta_{1} \Delta_{1}\right\rangle$ DUE TO MESOSCOPIC FLUCTUATIONS}

In this Section we evaluate the zero-momentum correlation function

$$
\Phi=\frac{\left\langle\tilde{\Delta}_{1}\left(T_{c}\right) \tilde{\Delta}_{1}\left(T_{c}\right)\right\rangle_{\mathbf{q}=0}}{\tilde{\Delta}\left(T_{c}\right) \tilde{\Delta}\left(T_{c}\right)}
$$

due to mesoscopic fluctuations of $F_{\text {dis }}$ and $\lambda_{\text {dis }}$.

\section{Correlation function $\left\langle F_{\text {dis }} F_{\text {dis }}\right\rangle$}

The correlator of Gorkov functions in the Matsubara representation is calculated with the help of imaginary-time replica sigma-model following the line of Ref. [S1]. The resulting expression has the form

$$
\left\langle F_{\mathrm{dis}}(\varepsilon, \mathbf{r}) F_{\mathrm{dis}}\left(\varepsilon^{\prime}, \mathbf{r}^{\prime}\right)\right\rangle=F_{0}(\varepsilon) F_{0}\left(\varepsilon^{\prime}\right) \frac{\left[\Pi_{\varepsilon \varepsilon^{\prime}}\left(\mathbf{r}, \mathbf{r}^{\prime}\right)\right]^{2}}{(\pi \nu)^{2}},
$$

where $\nu$ is the 2D one-particle DOS at the Fermi level (per single spin projection), and $\Pi$ is the diffusion operator on top of the superconducting state:

$$
\Pi_{\varepsilon \varepsilon^{\prime}}^{-1}=-D \nabla^{2}+\mathfrak{E}(\varepsilon)+\mathfrak{E}\left(\varepsilon^{\prime}\right),
$$


where

$$
\mathfrak{E}(\varepsilon)=\sqrt{\varepsilon^{2}+\tilde{\Delta}^{2}(\varepsilon)} .
$$

For the zero Fourier component we get

$$
\left\langle F_{\text {dis }}(\varepsilon) F_{\text {dis }}\left(\varepsilon^{\prime}\right)\right\rangle_{\mathbf{q}=0}=\frac{F_{0}(\varepsilon) F_{0}\left(\varepsilon^{\prime}\right)}{4 \pi^{3} \nu^{2} D} \frac{1}{\mathfrak{E}(\varepsilon)+\mathfrak{E}\left(\varepsilon^{\prime}\right)} .
$$

The corresponding contribution to Eq. (S25) has the form

$$
\Phi^{(F F)}=\frac{L_{0}^{2}}{\Delta^{4}(T)} \frac{(2 \pi T)^{4}}{4 \pi^{3} \nu^{2} D} \sum_{\varepsilon_{1}, \varepsilon_{2}, \varepsilon_{3}, \varepsilon_{4}>0} \lambda\left(\varepsilon_{1}, \varepsilon_{2}\right) \lambda\left(\varepsilon_{3}, \varepsilon_{4}\right) \frac{F_{0}\left(\varepsilon_{1}\right) F_{0}\left(\varepsilon_{2}\right) F_{0}\left(\varepsilon_{3}\right) F_{0}\left(\varepsilon_{4}\right)}{\mathfrak{E}\left(\varepsilon_{2}\right)+\mathfrak{E}\left(\varepsilon_{4}\right)} .
$$

This expression is typical to fluctuation contributions to $\Phi$. Among four energy summations, two are logarithmic involving large energies, $\varepsilon \gg T_{c}$, while the other two come from $\varepsilon \sim T_{c}$, where $\tilde{\Delta}(\varepsilon)$ can be approximated by $\Delta(T)$. The latter summations introduce the dimensionless function

$$
N\left(\frac{T}{T_{c}}\right)=16 T^{2} \sum_{\varepsilon_{1}, \varepsilon_{2}>0} \frac{\Delta(T)}{\mathfrak{E}_{1} \mathfrak{E}_{2}\left(\mathfrak{E}_{1}+\mathfrak{E}_{2}\right)}=\frac{2}{\pi} \int_{0}^{\infty} \frac{d \theta}{\cosh \theta} \tanh ^{2}\left[\frac{\Delta(T)}{2 T} \cosh \theta\right]=\left\{\begin{array}{cc}
\frac{14 \zeta(3)}{2 \pi^{3}} \frac{\Delta(T)}{T}, & T_{c}-T \ll T_{c} ; \\
1, & T \ll T_{c} .
\end{array}\right.
$$

Performing summations over $\varepsilon_{2}$ and $\varepsilon_{4}$ in Eq. (S30) we get

$$
\Phi^{(F F)}=\frac{1}{16 \pi \nu^{2} D \Delta^{3}(T)} L_{0}^{2}\left(\frac{T}{T_{c}}\right) N\left(\frac{T}{T_{c}}\right)\left(2 \pi T \sum_{\varepsilon>0} \lambda\left(\varepsilon, T_{c}\right) F_{0}(\varepsilon)\right)^{2}
$$

Summation is done with the help of the SCE (4), and we obtain finally

$$
\Phi^{(F F)}=\frac{1}{16 \pi \nu^{2} D \Delta(T)} L_{0}^{2}\left(\frac{T}{T_{c}}\right) N\left(\frac{T}{T_{c}}\right)
$$

\section{Correlation function $\left\langle\delta \lambda_{\text {dis }} \delta \lambda_{\text {dis }}\right\rangle$}

Mesoscopic fluctuations of the return probability which determines $\delta \lambda\left(\varepsilon, \varepsilon^{\prime}\right)=-\lambda_{g}^{2} \ln \left[1 / \max \left(\varepsilon, \varepsilon^{\prime}\right) \tau\right]$ have been calculated for the normal state in Ref. [S2]:

$$
\left\langle\lambda_{\text {dis }}\left(\varepsilon_{1}, \varepsilon_{2}\right) \lambda_{\text {dis }}\left(\varepsilon_{3}, \varepsilon_{4}\right)\right\rangle_{\mathbf{q}=0}=\frac{\delta \lambda\left(\varepsilon_{1}, \varepsilon_{2}\right) \delta \lambda\left(\varepsilon_{3}, \varepsilon_{4}\right)}{16 \pi^{3} \nu^{2} D}\left(\frac{1}{\left|\varepsilon_{13}\right|}+\frac{1}{\left|\varepsilon_{14}\right|}+\frac{1}{\left|\varepsilon_{23}\right|}+\frac{1}{\left|\varepsilon_{24}\right|}\right),
$$

where $\varepsilon_{i j} \equiv \varepsilon_{i}+\varepsilon_{j}$.

Generalization to the superconducting case is achieved by replacing $\varepsilon \rightarrow \mathfrak{E}(\varepsilon)$. All four terms in Eq. (S34) equally contribute to $\Phi$, and we get

$$
\Phi^{(\lambda \lambda)}=\frac{L_{0}^{2}}{\Delta^{4}(T)} \frac{(2 \pi T)^{4}}{4 \pi^{3} \nu^{2} D} \sum_{\varepsilon_{1}, \varepsilon_{2}, \varepsilon_{3}, \varepsilon_{4}>0} \delta \lambda\left(\varepsilon_{1}, \varepsilon_{2}\right) \delta \lambda\left(\varepsilon_{3}, \varepsilon_{4}\right) \frac{F_{0}\left(\varepsilon_{1}\right) F_{0}\left(\varepsilon_{2}\right) F_{0}\left(\varepsilon_{3}\right) F_{0}\left(\varepsilon_{4}\right)}{\mathfrak{E}\left(\varepsilon_{2}\right)+\mathfrak{E}\left(\varepsilon_{4}\right)} .
$$

Analogously to Eq. (S30)], summations over $\varepsilon_{2}$ and $\varepsilon_{4}$ yields $N\left(T / T_{c}\right)$, while summations over $\varepsilon_{1}$ and $\varepsilon_{3}$ are converted to a logarithmic integral:

$$
\Phi^{(\lambda \lambda)}=\frac{1}{16 \pi \nu^{2} D \Delta(T)} L_{0}^{2}\left(\frac{T}{T_{c}}\right) N\left(\frac{T}{T_{c}}\right)\left(\int_{0}^{\zeta_{*}} \delta \lambda\left(\zeta, \zeta_{*}\right) \cosh \lambda_{g}\left(\zeta_{*}-\zeta\right) d \zeta\right)^{2}
$$

and hence

$$
\Phi^{(\lambda \lambda)}=\frac{1}{16 \pi \nu^{2} D \Delta(T)} L_{0}^{2}\left(\frac{T}{T_{c}}\right) N\left(\frac{T}{T_{c}}\right)\left[\cosh \lambda_{g} \zeta_{*}-1\right]^{2}
$$




\section{Correlation function $\left\langle F_{\mathrm{dis}} \delta \lambda_{\mathrm{dis}}\right\rangle$}

The cross term is evaluated analogously:

$$
\Phi^{(F \lambda)}=-2 \frac{1}{16 \pi \nu^{2} D \Delta(T)} L_{0}^{2}\left(\frac{T}{T_{c}}\right) N\left(\frac{T}{T_{c}}\right)\left[\cosh \lambda_{g} \zeta_{*}-1\right] .
$$

\section{Resulting expression for $\left\langle\Delta_{1} \Delta_{1}\right\rangle$}

Adding (S33), (S37) and (S38), we obtain

$$
\Phi=\frac{1}{16 \pi \nu^{2} D \Delta(T)} K\left(\frac{T}{T_{c}}\right) \cosh ^{2} \lambda_{g} \zeta_{*}=\frac{\pi D}{g\left(g-g_{c}\right) \Delta(T)} K\left(\frac{T}{T_{c}}\right),
$$

where we have introduced $K\left(T / T_{c}\right)=L_{0}^{2}\left(T / T_{c}\right) N\left(T / T_{c}\right)$, used the relation $g=4 \pi \nu D$, and employed $\cosh ^{2} \lambda_{g} \zeta_{*}=$ $g /\left(g-g_{c}\right)$. With the help of Eq. (S25), one readily obtains Eq. (10) of the main text.

It's worth noting that Eq. (S39) agrees with our previous results [S2]. Indeed, in the limit $T \rightarrow T_{c}$, Eq. (S39) can be simplified with the help of Eqs. (S23) and (S31) as

$$
\langle\langle\Delta(T) \Delta(T)\rangle\rangle_{\mathbf{q}=0}=\frac{2 T^{3}}{7 \zeta(3) \nu^{2} D \Delta^{2}(T)} \cosh ^{2} \lambda_{g} \zeta_{*} .
$$

On the other hand, the same correlator can be obtained from the correlation function of the coefficient $\alpha$ in the Ginzburg-Landau (GL) expansion [S2]:

$$
\langle\langle\alpha \alpha\rangle\rangle_{\mathbf{q}=0}=\frac{7 \zeta(3)}{8 \pi^{4} D T} \cosh ^{2} \lambda_{g} \zeta_{*}
$$

with the help of the relation

$$
\langle\langle\Delta(T) \Delta(T)\rangle\rangle_{\mathbf{q}=0}=\frac{\langle\langle\alpha \alpha\rangle\rangle_{\mathbf{q}=0}}{4 \beta^{2} \Delta^{2}(T)}
$$

where $\beta=7 \zeta(3) \nu /\left(8 \pi^{2} T^{2}\right)$ is the nonlinear coefficient in the GL functional. One can easily verify that Eq. (S42) coincides with Eq. (S40).

\section{DENSITY OF THE SUBGAP STATES}

Introducing $\theta=\pi / 2+i \psi$, we rewrite Eq. (12) as

$$
-\xi^{2} \nabla^{2} \psi+F(\psi)=-\frac{\delta \Delta(\mathbf{r}) \sinh \psi}{\Delta_{0}},
$$

where $\xi^{2}=D / 2 \Delta_{0}$, and

$$
F(\psi)=-\frac{E}{\Delta_{0}} \cosh \psi+\sinh \psi-\eta \sinh \psi \cosh \psi
$$

At the minigap $\left(E=E_{g}\right), \cosh \psi_{g}=\eta^{-1 / 3}$. For small deviation from the gap, the function $F(\psi)$ in the vicinity of its maximum can be written as

$$
F(\psi) \approx \Omega\left(\psi-\psi_{0}\right)-\rho\left(\psi-\psi_{0}\right)^{2}
$$

where

$$
\Omega=\sqrt{6} \sqrt[4]{1-\eta^{2 / 3}} \sqrt{\frac{E_{g}-E}{\Delta_{0}}}, \quad \rho=\frac{3}{2} \eta^{1 / 3} \sqrt{1-\eta^{2 / 3}}
$$


Comparison of the linear in $\psi$ terms in the left-hand side of Eq.

$$
L_{E}=\frac{\xi}{\sqrt{\Omega}} .
$$

At the mean-field level, $\psi$ is real below the gap. Finite DOS corresponds to appearance of a nonzero $\operatorname{Im} \psi$ due to a large negative fluctuation of $\delta \Delta(\mathbf{r})$. Its probability is given by

$$
\mathcal{P} \propto \exp \left(-\frac{1}{2 f(0)} \int \delta \Delta^{2}(\mathbf{r}) d^{d} r\right) \equiv e^{-S_{d}},
$$

where we have used that the instanton size, $L_{E}$, exceeds the correlation length, $\xi_{0}$, of the order parameter fluctuations. At the quantitative level, the instanton action $S$ can be estimated as follows. To produce a nonzero DOS at $E<E_{g}$, the optimal fluctuation of $\delta \Delta(\mathbf{r})$ should have the magnitude of $-\left(E_{g}-E\right)$ and the spacial extent of $L_{E}$, which immediately gives the estimate [S3]

$$
S_{d} \sim \frac{\Delta_{0}^{2} \xi^{d}}{f(0)}\left(\frac{E_{g}-E}{E_{g}}\right)^{2-d / 4}
$$

To find the numerical coefficient in Eq. (S49), one has to solve the instanton equation. Measuring coordinates in terms of $L_{E}$ introduced in Eq. (S47), appropriately rescaling $\psi-\psi_{0}$, and replacing $\sinh \psi$ in the right-hand side of Eq. (S43) by its value at $E_{g}$, we rewrite Eq. (S43) in a universal dimensionless form:

$$
-\nabla^{2} \phi+\phi-\phi^{2}=h(\mathbf{r})
$$

where

$$
\delta \Delta(\mathbf{r})=-\frac{\Delta_{0} \Omega^{2}}{\rho \sinh \psi_{g}} h\left(\mathbf{r} / L_{E}\right) .
$$

Minimization of the functional $\int h^{2}(\mathbf{r}) d^{d} r$ leads to the fourth order differential equation for $\phi(\mathbf{r})$ :

$$
\left(-\nabla^{2}+1-2 \phi\right)\left(-\nabla^{2} \phi+\phi-\phi^{2}\right)=0 .
$$

The spherically symmetric optimal fluctuation solving (S52) in $d$ dimensions satisfies the second-order differential equation $[\mathrm{S} 4]$

$$
-\nabla_{d-2}^{2} \phi+\phi-\phi^{2}=0
$$

where $\nabla_{d-2}^{2} \equiv \partial^{2} / \partial r^{2}-(d-3) r^{-1} \partial / \partial r$ is the radial part of the Laplace operator in the $(d-2)$-dimensional space. The instanton is characterized by the number

$$
a_{d}=\int h^{2} d^{d} r=4 \int \frac{\phi^{\prime 2}}{r^{2}} d^{d} r= \begin{cases}48 \pi / 5, & d=3, \\ 4.1, & d=2,\end{cases}
$$

where $a_{3}$ follows from the exact solution $\phi_{3}(r)=(3 / 2) \cosh ^{-2}(r / 2)$ [S3], while $a_{2}$ is obtained by a numerical solution of Eq. (S53).

Returning to the dimensional variables, we get for the instanton action in the limit $\eta \ll 1$ :

$$
S_{d}=\frac{8 a_{d}}{6^{d / 4}} \frac{\Delta_{0}^{2} \xi^{d}}{f(0)}\left(\frac{E_{g}-E}{E_{g}}\right)^{2-d / 4} .
$$

In the $2 \mathrm{D}$ case,

$$
S_{2}=\frac{4 a_{2}}{\sqrt{6}} \frac{D \Delta_{0}}{f(0)}\left(\frac{E_{g}-E}{E_{g}}\right)^{3 / 2}
$$

leading to Eqs. (18) and (19). 


\section{ROLE OF A FINITE FILM THICKNESS}

In films with a finite thickness $d \lesssim \xi_{0}$, there exists a contribution to the depairing parameter $\eta$ in Eq. (13) coming from large wave vectors $(q d \gg 1)$, where diffusion is three-dimensional:

$$
\eta_{3 \mathrm{D}}=\frac{2}{\Delta} \int \frac{f(\mathbf{q})}{D q^{2}} \frac{d^{3} \mathbf{q}}{(2 \pi)^{3}}=\frac{1}{\pi^{2} \Delta D} \int_{1 / d}^{1 / l} f(q) d q
$$

[here $\Delta \equiv \Delta(T)$ is the temperature-dependent BCS order parameter]. In this region, Coulomb effects are weak and all complications related with the energy dependence of $\lambda$ and $\Delta$ can be neglected. Equation (8) is then replaced by a simpler expression written for an arbitrary 3D wave vector:

$$
\delta \Delta(T, \mathbf{q})=L_{q}\left(\frac{T}{T_{c}}\right) 2 \pi T \sum_{\varepsilon>0} F_{\mathrm{dis}}(\varepsilon, \mathbf{q})
$$

where $L_{q}\left(T / T_{c}\right)$ is the BCS fluctuation propagator at finite momentum:

$$
L_{q}^{-1}\left(T / T_{c}\right)=2 \pi T \sum_{\varepsilon>0} \frac{\mathfrak{E}(\varepsilon) D q^{2}+2 \Delta^{2}(T)}{\mathfrak{E}^{2}(\varepsilon)\left[D q^{2}+2 \mathfrak{E}(\varepsilon)\right]} .
$$

In the limit $q \xi_{0} \gg 1$, one recovers the known inverse logarithmic decay of the fluctuation propagator [S3]:

$$
L_{q}\left(T / T_{c}\right) \approx \frac{1}{\ln \left(D q^{2} / T_{c}\right)}, \quad q \xi_{0} \gg 1
$$

With the help of Eq. ([S26) the correlation function $f(\mathbf{q})=\langle\delta \Delta \delta \Delta\rangle_{\mathbf{q}}$ can be written as

$$
f(\mathbf{q})=L_{q}^{2}\left(\frac{T}{T_{c}}\right)\left(\frac{T \Delta}{\nu_{3}}\right)^{2} \sum_{\varepsilon_{1}, \varepsilon_{2}>0} \int \frac{d^{3} \mathbf{k}}{(2 \pi)^{3}} \frac{\Pi_{\varepsilon \varepsilon^{\prime}}(\mathbf{k}+\mathbf{q} / 2) \Pi_{\varepsilon \varepsilon^{\prime}}(\mathbf{k}-\mathbf{q} / 2)}{\mathfrak{E}\left(\varepsilon_{1}\right) \mathfrak{E}\left(\varepsilon_{2}\right)},
$$

where $\nu_{3}=\nu / d$ is the 3D DOS at the Fermi level. Integrating over $\mathbf{k}$ with the help of the Feynman's trick we get

$$
f(\mathbf{q})=\frac{1}{8 \pi} L_{q}^{2}\left(\frac{T}{T_{c}}\right)\left(\frac{T \Delta}{\nu_{3} D}\right)^{2} \sum_{\varepsilon_{1}, \varepsilon_{2}>0} \frac{1}{\mathfrak{E}\left(\varepsilon_{1}\right) \mathfrak{E}\left(\varepsilon_{2}\right)} \int_{0}^{1} \frac{d x}{\left[x(1-x) q^{2}+\left(\mathfrak{E}\left(\varepsilon_{1}\right)+\mathfrak{E}\left(\varepsilon_{2}\right)\right) / D\right]^{1 / 2}} .
$$

In the limit $q \xi_{0} \gg 1$, only large $\varepsilon_{1,2} \gg T_{c}$ with $\mathfrak{E}(\varepsilon) \approx|\varepsilon|$ are important. Replacing summations by integrations and using Eq. (S60) we find

$$
f(\mathbf{q})=\frac{1}{8 \pi^{3}} L_{q}^{2}\left(\frac{T}{T_{c}}\right)\left(\frac{\Delta}{\nu_{3} D}\right)^{2} \int_{T_{c}}^{D q^{2}} \frac{d \varepsilon_{1}}{\varepsilon_{1}} \frac{d \varepsilon_{2}}{\varepsilon_{2}} \int_{0}^{1} \frac{d x}{\left[x(1-x) q^{2}+\left(\varepsilon_{1}+\varepsilon_{2}\right) / D\right]^{1 / 2}} \approx \frac{1}{8 \pi^{2} q}\left(\frac{\Delta}{\nu_{3} D}\right)^{2}
$$

The obtained $1 / q$ dependence of the correlation function $f(q)$ leads in Eq. S64 to the logarithmic contribution from the region $l \ll r \ll d$ :

$$
\eta_{3 \mathrm{D}}=\frac{\Delta(T)}{8 \pi^{4} D}\left(\frac{1}{\nu_{3} D}\right)^{2} \ln \frac{d}{l}
$$

leading to Eq. (17).

For even thicker films with $d \gg \xi_{0}$, the 2D contribution (15) is small and Eq. (S64) with $d$ replaced by $\xi_{0}$ gives the leading contribution to the depairing rate.

[S1] M. Houzet and M. A. Skvortsov, Phys. Rev. B 77, 057002 (2008).

[S2] M. A. Skvortsov and M. V. Feigel'man, Phys. Rev. Lett. 95, 057002 (2005).

[S3] A. I. Larkin and Yu. N. Ovchinnikov, Zh. Eksp. Teor. Fiz. 61, 2147 (1971) [Sov. Phys. JETP 34, 1144 (1972)].

[S4] A. Silva and L. B. Ioffe, Phys. Rev. B 71, 104502 (2005). 